Case Report

\title{
Anal-sphincter Disruption after Obstetric Fourth-degree Laceration-report of a Case
}

\author{
Naokuni YASUdA ${ }^{1}$, Makoto W $_{\text {ATANABE }}{ }^{1)}$, Takahiro TANAKA ${ }^{1}$, \\ Akira TsunOdA $^{2)}$ and Mitsuo KuSANO ${ }^{2)}$
}

\begin{abstract}
A 31-year-old woman was admitted to the hospital because of soiling and anal pain. She had a normal delivery on the 3 rd of August 2000 and a mediolateral episiotomy was performed. Fourth-degree laceration was identified at delivery and a gynecologist performed the primary suture. Surgery was carried out on the 12th of March 2001 after recovery from inflammation at the tear site. Anal ultrasound examination revealed a defect of the anterior external sphincter muscle. The sphincter, levator ani, anal canal epithelium and vaginal mucosa were repaired. She has been well, with no evidence of recurrence, for 10 months. Eight months after surgery, she was able to recommence a normal sexual life both physically and psychologically. A manometric study performed 5 months after surgery revealed that maximum squeeze pressure had increased 2.5 fold. Success in treatment of patients with tears should be measured not only in terms of successful long-term closure but also in terms of the patient's satisfaction with postoperative incontinence and sexual life.
\end{abstract}

Key words : anal-sphincter disruption, fourth-degree laceration, obstetric trauma

\section{Introduction}

Fecal incontinence can be caused by trauma, colorectal disease, congenital anomaly, neurological disease and other miscellaneous causes. Most obstetric traumas following delivery are recognized and repaired by the obstetrician, including fourth-degree perineal lacerations. The formation of sepsis or hematoma after primary repair can lead to suture breakage and fecal incontinence.

\section{Case Report}

A 31-year-old Japanese woman was admitted to the hospital because of soiling and anal pain. She had a normal delivery on the 3rd of August 2000. Mediolateral episiotomy was carried out and fourth-degree laceration was identified at delivery. A gynecologist performed the primary suture, and follow up treatment included intravenous hyperalimentation. Ten days later, the suture broke down due to inflammation. Anal pain and soiling developed. She was referred to our department on the 4th of September (Fig. 1). The vaginal septum was disrupted from the vaginal opening to above the dentate line. Both edges of the walls were severely inflamed, and a foul-smelling vaginal discharge was seen. There was no

\footnotetext{
1) Department of Surgery, Isesaki Municipal Hospital, 1180 Tsunatori-Town Isesaki 372-0812, Japan.

${ }^{2)}$ Second Department of Surgery, Showa University
} 


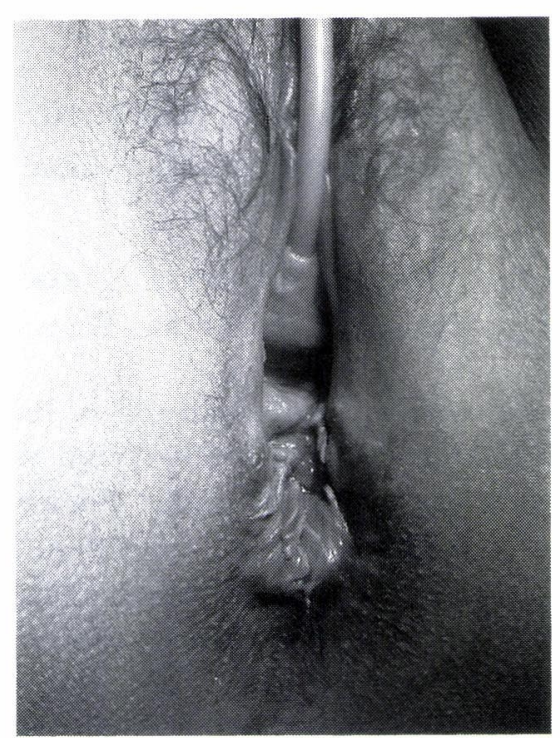

Fig. 1. Preoperative perineal appearance of the patient. The anal sphincter was disrupted due to misrepaired fourth-degree laceration.

fistula formation. She had liquid meals for 8 days to reduce anal pain and inflammation at the tear site. After discharge, she needed a pad due to soiling, and her bowel movement was twice a day.

Before surgery, a manometric study was performed using a three-channel catheter (G 92 -005/485, Synectics Medical, Stockholm, Sweden), a PC Polygraph HR (Medtronic Functional Diagnostics, Skovlunde, Denmark) and a personal computer with Polygram Software (Medtronic Functional Diagnostics). The study was performed in the left lateral decubitus position. Bowel preparation was not necessary before the study. Maximum basal pressure and maximum squeeze pressure were measured using a station pull-through technique. Low maximum squeeze pressure indicated the presence of a sphincter injury (Table 1). Surgery was carried out on the 12th of March 2001 after reduction of inflammation at the tear (Fig. 2). Polyethylene glicholelectrolite was used for bowel preparation. Under general anesthesia, with the patient in the lithotomy position (Fig. 1), anal ultrasound examination revealed a defect in the anterior external sphincter muscle. Radial incisions were made to dissect the vaginal mucosa, and to identify anal sphincter muscles. Adrenaline ( 1 in 200,000) was injected into the submucosal layer. For sphincter repair, three horizontal mattress sutures of vicryl were inserted to bring the edges of the anal sphincter muscles together (Fig. 2). Levator ani, anal canal epithelium and vaginal mucosa were repaired respectively. The central incision was left for drainage (Fig. 3). On the second day following the operation we commenced washing of the wound. She was able to have solid meals and passed a stool on the forth day following the operation. Antibiotics were given intravenously for four days. She was discharged on the twenty-fourth day following the operation. A manometric study was performed 5 months after surgery. Maximum basal pressure had increased slightly, and maximum squeeze pressure had 
Table 1. Results of a manometric study before and after surgery

\begin{tabular}{lcc}
\hline & pre-operation & post-operation \\
\hline Maximum basal pressure $(\mathrm{mmHg})$ & 40 & 50 \\
Maximum squeeze pressure $(\mathrm{mmHg})$ & 60 & 150 \\
Maximum tolerable volume $(\mathrm{ml})$ & 300 & 250
\end{tabular}

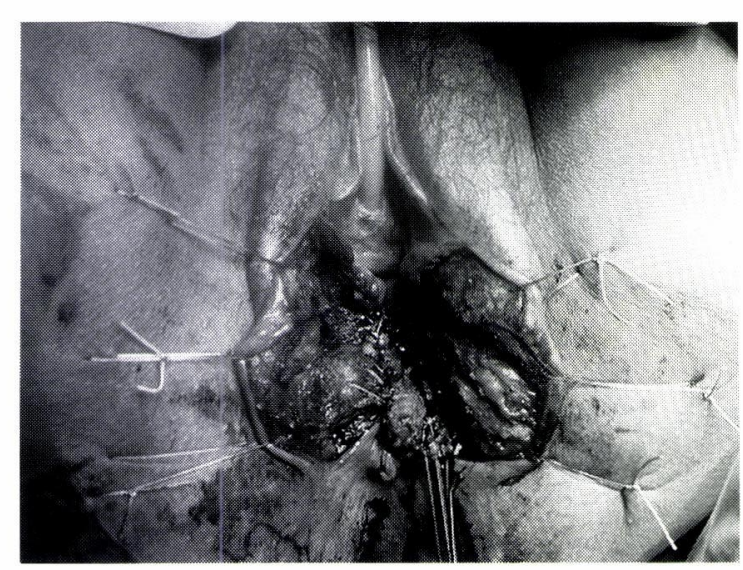

Fig. 2. Horizontal mattress sutures were used to perform overlapping sphincteroplasty.

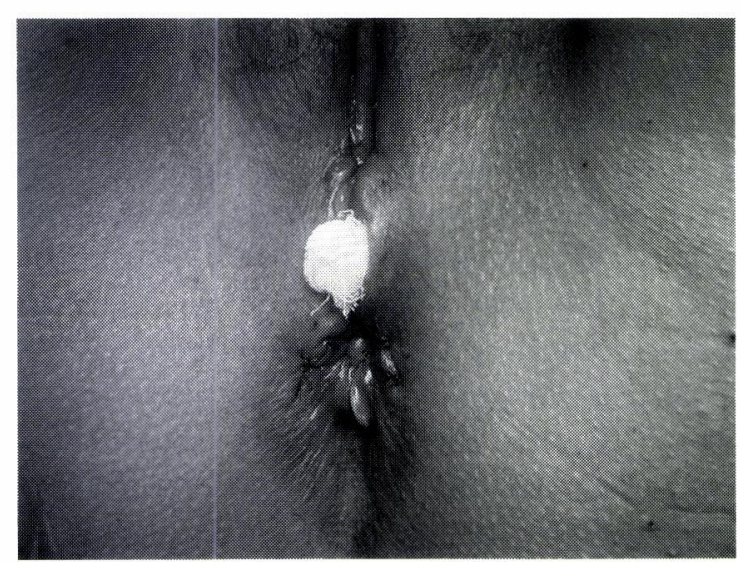

Fig. 3. Postoperative perineal appearance of the patient. The skin is left open for drainage.

increased 2.5 fold since surgery (Table 1). She has been well with no evidence of recurrence for 10 months. Eight months after surgery, she was able to recommence a normal sexual life both physically and psychologically (Fig. 4). 


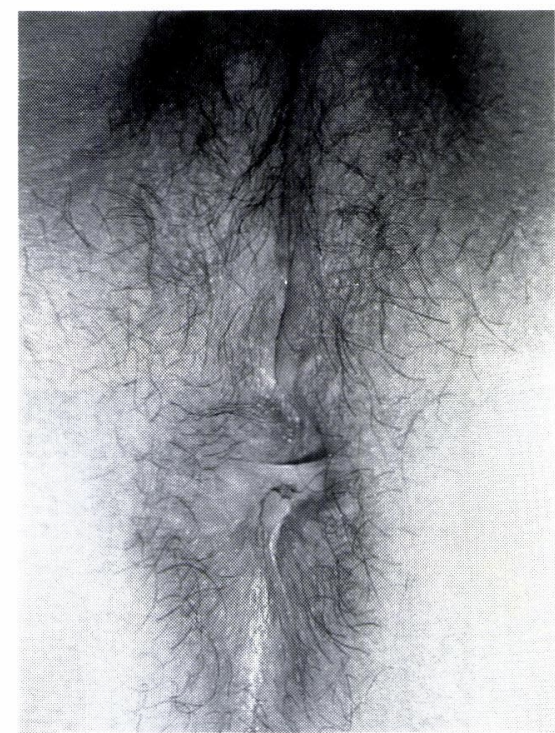

Fig. 4. Clinical finding eight months after surgery shows the repaired vaginal septum.

\section{Discussion}

The incidence of anorectal complications following vaginal delivery is approximately $5 \%$ and $0.1 \%$ of vaginal deliveries result in rectovaginal fistulas ${ }^{1)}$. The causes of fecal incontinence following vaginal delivery include obstetric trauma or sphincter atrophy following pudendal nerve damage ${ }^{2-4}$. There is no indisputable evidence that episiotomy protects women from pelvic stress, although numerous studies suggest that midline episiotomy carries a higher risk of injury than mediolateral episiotomy ${ }^{5,6)}$. Corman ${ }^{7)}$ suggests that an anterior located anus (ectopic anus) is a risk factor. It is important that obstetricians be wary of performing an episiotomy if the anus is anteriorly displaced toward the vagina. Rectovaginal fistulas may arise from an inadequately repaired fourth-degree perineal tear, postoperative infection, or ischemic necrosis of the rectovaginal septum due to prolonged labor ${ }^{3,8)}$. In this case, the patient had her first vaginal delivery without vacuum or forceps. The tear was repaired at the time of delivery and its recurrence following the operation may have been due to inadequate repair or postoperative infection.

The most common symptoms are passage of flatus and feces from the vagina ${ }^{3)}$. Most patients with these symptoms are socially disabled and some desire to have more children. Consideration of a patient's social problems pre and/or post-operation should be taken into account.

In terms of management of obstetric injuries, the timing of repair is crucial. A 4-6 month time period may be necessary to allow reduction of local tissue inflammation ${ }^{3,8)}$. Another reason for waiting 3-6 months is the possibility of spontaneous closure ${ }^{8)}$. Some authors $^{8)}$ believe that a diverting colostomy is not necessary. For patients who have had several failed repairs or severe inflammation, a diverting colostomy may be useful ${ }^{3)}$. We did not perform a diverting colostomy in this case due to her financial position and the fact 
that as a young woman she would have experienced cosmetic stress.

A history of previous repairs decreases the chance of success due to poor blood supply in the vicinity of scar tissue ${ }^{8)}$. Multiple repeated fistulation may lead to permanent stoma. More advanced techniques such as gracilis muscle interposition, an artificial anal sphincter, should be considered, although the long-term success of this technique is unknown. There are three approaches to local repair including the transanal approach, the vaginal approach and the perineal approach. In this case, we performed sphincteroplasty as a perineal approach, because the anal ultrasound taken during surgery showed a sphincter defect. Successful repair was indicated by postoperative anal continence and manometric results showing increased maximum squeeze pressure following surgery. Although physically capable, it took her 8 months to return to normal sexual activity because psychologically she was fearful that penetration might cause a recurrent tear.

Success in treatment of patients with tears should be measured not only in terms of successful long-term closure but also in terms of the patient's satisfaction with postoperative incontinence and sexual life.

\section{References}

1) Venkatesh KS, Ramanujam PS, Larson DM and Haywood MA : Anorectal complications of vaginal delivery. Dis Colon Rectum 32 : 1039-1041 (1989)

2) Sultan AH, Kamm MA, Hudson CN, Thomas JM and Bartram CI : Anal-sphincter disruption during vaginal delivery. N Engl J Med 329 : 1905-1911 (1993)

3) Tsang CBS and Rothenberger DA : Rectovaginal fistulas. Surg Clin North Am 77 : 95-114 (1997)

4) Belmont-Montes C, Hagerman G, Vega-Yepes PA, Hernandez-de-Anda E and Fonseca-Morales V: Anal sphincter injury after vaginal delivery in primiparous females. Dis Colon Rectum 44 : 1244-1248 (2001)

5) Henriksen TB, Bek KM, Hedegaard $M$ and Secher NJ : Episiotomy and perineal lesions in spontaneous vaginal deliveries. Br J Obstet Gynaecol 99 : 950-954 (1992)

6) Peleg D, Kennedy CM, Merrill D and Zlatnik FJ : Risk of repetition of a severe perineal laceration. Obstet Gynecol 93 : 1021-1024 (1999)

7) Corman ML: Anal incontinence. In : Colon and Rectal Surgery Third edition J.B. Lippincott Company, Philadelphia pp 188-261 (1993)

8) Lowry AC, Thorson AG, Rothenberger DA and Goldberg SM: Repair of simple rectovaginal fistula. Dis Colon Rectum 31 : 676-678 (1988)

[Received June 4, 2002 : Accepted June 28, 2002] 